\title{
Alelopatia intra-específica de extratos aquosos de folhas e raízes de alfafa na germinação e no crescimento inicial de plântulas de dois materiais de alfafa: crioulo e melhorado
}

\author{
Intra-specific allelopathy of leaves and roots aqueous extracts on germination \\ and early seedling growth of two alfalfa materials: crioulo and improved
}

Betânia Fraga Pereira ${ }^{\mathrm{I}}$ André Fischer Sbrissia' ${ }^{\mathrm{II}}$ Beatriz Monte Serrat ${ }^{\mathrm{II}}$

\begin{abstract}
A alfafa é uma das culturas conhecidas que RESUMO apresentam um tipo específico de alelopatia denominado autotoxicidade. Dessa forma, o objetivo deste trabalho foi avaliar os efeitos da concentração de extratos aquosos de partes (folhas e raízes) da planta de alfafa (material Crioulo) na germinação e no crescimento inicial de plântulas de dois materiais de alfafa (Crioulo e P5454). Placas de Petri contendo 50 sementes de cada variedade foram umedecidas com $10 \mathrm{ml}$ dos extratos em duas concentrações: $50 \%$ e $100 \%$ (extrato puro). Água deionizada foi usada como controle. $O$ delineamento experimental foi inteiramente casualizado, com cinco repetições para cada variedade. Após seis dias da germinação, procedeu-se à contagem do número de sementes germinadas, bem como à medição do comprimento do hipocótilo e da radícula de cada plântula. Os extratos aquosos das folhas inibiram a germinação e o comprimento da radícula e do hipocótilo das duas variedades. No entanto, para o material Crioulo, a inibição na germinação e no desenvolvimento inicial só ocorreu com o uso do extrato puro das folhas, enquanto que na $\mathrm{P} 5454$ o extrato diluído a 50\% já reduziu tanto a germinação quanto o comprimento das plântulas. Os extratos aquosos das raízes não causaram inibição na germinação nem no comprimento das plântulas. Os resultados sugerem variações intra-específicas na tolerância aos compostos aleloquímicos e uma produção preferencial dos mesmos na parte aérea da planta de alfafa, material Crioulo.
\end{abstract}

Palavras-chave: autoxicidade, Medicago sativa, alelopatia intra-específica.

\section{ABSTRACT}

Alfalfa is one of the known crops that present a specific type of allelopathy called autotoxicity. Hence, this research was aimed at evaluating the concentration effects of aqueous extracts of plant parts (leaves and roots) of alfalfa (var. Crioula) on germination and early development of seedlings of two alfalfa (Crioula and P5454) varieties. Fifty seed of each variety were placed in Petri dishes with moistened filter paper. Aliquots (ten $\mathrm{ml}$ ) from each part extracts at two concentrations, 50 or $100 \%$ (pure extract), were added to petri dishes, and distilled water was used as a control. Treatments placed in Petri dishes were randomly arranged in a completely randomized design with five replicates. Measurements included: germination, and radicle and hypocotyl lengths taken six days after germination. The aqueous extracts of leaves inhibited germination and radicle and hypocotyl length of the two alfalfa varieties. However, on the var. Crioula germination and early development was inhibited only when it was used pure extracts of leaves, while in var. P5454 leaves extracts diluted to 50\% already had some reduction on germination and seedlings length. The aqueous extracts of the alfalfa roots did not cause inhibition on germination and seedlings lengths. The results suggest intra-specific variations on tolerance to the allelochemicals and a preferential production of them in the aerial part of the alfalfa plant, var. Crioula.

Key words: Medicago sativa, intra-specific allelopathy.

A autoxicidade é uma forma específica de alelopatia na qual a planta produz compostos químicos que prejudicam a germinação e/ou o desenvolvimento da própria espécie (CHON, 2004). A autoxicidade em alfafa (Medicago sativa L.) foi primeiramente verificada por JENSEN et al. (1981) e normalmente ocorre quando se tenta semear alfafa sobre uma cultura anterior de alfafa (JENNINGS \& NELSON, 2002a), ou ainda quando se faz a semeadura em espaços vazios de estandes

IEmbrapa Clima Temperado, BR 392, km 78, 96001-970, Pelotas, RS, Brasil.

IDepartamento de Zootecnia, Universidade do Estado de Santa Catarina (UDESC). Av. Luiz de Camões, 2090, 88520-000, Lages, SC, Brasil. E-mail: afsbrissia@cav.udesc.br. Autor para correspondência.

IIIDepartamento de Solos, Universidade Federal do Paraná (UFPR). Curitiba, PR, Brasil. 
com baixa população de plantas (JENNINGS \& NELSON, 2002b). De acordo com CHON et al. (2006), muitas vezes é necessário esperar até dois anos para que se possa implantar uma cultura de alfafa em locais onde existia a cultura. As plantas têm capacidade de produzir aleloquímicos em todos os seus órgãos, mas a concentração nos tecidos depende de diversos fatores, como solo, temperatura e pluviosidade. CHUNG \& MILLER, (1995), em estudo comparativo do efeito do extrato de diversas partes da planta de alfafa sobre a germinação e o desenvolvimento de plântulas da mesma, concluíram que os extratos das folhas e flores da alfafa foram os que apresentaram o maior efeito inibidor sobre a cultura.

O crescimento da raiz é mais sensível aos efeitos fitotóxicos dos compostos em baixas concentrações do que a germinação ou o crescimento do hipocótilo (CHON et al., 2003). Já em concentrações moderadas, ocorre um atraso na germinação e, em concentrações altas, pode ocorrer inibição significativa tanto no comprimento do hipocótilo quanto da radícula (MILLER, 1996). Apesar de a autotoxicidade ser amplamente descrita para alfafa, XUAN \& TSUZUKI (2002) verificaram diferenças varietais no poder fitotóxico, sugerindo que o potencial alelopático poderia estar relacionado com aspectos genéticos. Além disso, variações inter e intra-específicas no potencial alelopático de extratos de partes de plantas não são exclusividade de alfafa. PUTNAM \& DUKE (1974) e KONO et al. (1999), trabalhando com pepino (Cucumis sativus L.) e cevada (Hordeum vulgare L.), respectivamente, também observaram diferenças intraespecíficas na atividade alelopática.

O Brasil possui aproximadamente 26mil hectares de área plantada com alfafa (RASSINI et al., 2003), e, de acordo com os mesmos autores, a cultura vem despertando interesse crescente do setor produtivo, principalmente àqueles setores associados com a implantação de sistemas intensivos de produção de bovinos de corte e leite. Um dos materiais mais adaptados às condições nacionais é o Crioulo, fruto de um processo de seleção natural ocorrido na Região Sul do País (NUERNBERG, 1994). Dessa forma, o presente trabalho teve por objetivo verificar o potencial alelopático de extratos aquosos de folhas e raízes de alfafa, material Crioulo, na germinação e no desenvolvimento inicial de plântulas de dois materiais de alfafa, o próprio Crioulo e uma variedade híbrida, P5454.

A ação alelopática de extratos aquosos de alfafa (material Crioulo) foi avaliada por meio de testes de germinação e crescimento inicial de plântulas de alfafa de duas origens: um material Crioulo e uma variedade melhorada: P5454. Para tanto, plantas de alfafa (material Crioulo) foram coletadas no estádio vegetativo, procedentes de Piraquara, PR. As plantas coletadas foram separadas em folhas e raízes, lavadas e secadas ao ar. Para o preparo dos extratos, foram pesados $20 \mathrm{~g}$ de cada componente (folhas e raízes) do material seco, adicionados a 100ml de água deionizada e triturados em liqüidificador por dez minutos. Em seguida, filtrou-se o extrato em papel filtro INLAB tipo $10-11 \mathrm{~cm}$, centrifugando-o por quatro horas a 3500RPM, em centrífuga ECCO. O extrato utilizado foi o sobrenadante límpido obtido após a centrifugação, o qual foi mantido em geladeira $\left(10^{\circ} \mathrm{C}\right)$ até a sua utilização.

Para verificar o efeito desses extratos, testes de germinação foram conduzidos da seguinte forma: 50 sementes de alfafa de cada cultivar foram desinfetadas usando-se $5 \mathrm{ml}$ de hipoclorito de sódio em 95ml de água deionizada por cinco minutos, sendo posteriormente lavadas várias vezes com água deionizada (CHUNG \& MILLER, 1995). Após a desinfecção, as sementes do material Crioulo e P5454 foram colocadas em placas de Petri, previamente esterilizadas em autoclave e preparadas com papel filtro e algodão. Separadamente, alíquotas de $10 \mathrm{ml}$ de cada extrato, puro e diluído, foram adicionadas por placa de Petri, e água deionizada foi usada como controle. Dessa forma, os tratamentos experimentais foram constituídos de extratos aquosos utilizados de forma separada de duas partes da planta (folhas e raízes) em duas concentrações: $50 \%$ (extratos obtidos diluídos pela metade) e $100 \%$ (extrato puro) e um controle. Assim, o experimento apresentou três tratamentos para cada variedade, sendo realizado segundo um delineamento inteiramente casualizado, com cinco repetições, totalizando 25 observações para cada variedade. As placas de Petri foram mantidas em germinador tipo “Mangelsdorf”, com temperatura constante de $25^{\circ} \mathrm{C}$ e luminosidade controlada, no Laboratório de Sementes do Departamento de Fitotecnia da UFPR. Aos seis dias após a germinação, procedeu-se à contagem das sementes germinadas e determinou-se os comprimentos do hipocótilo e da radícula.

Uma análise de variância simples foi realizada para cada cultivar, utilizando-se o pacote estatístico MINITAB. Utilizou-se o teste de Tukey para comparação de médias entre tratamentos e assumiu-se um nível de significância de 5\%. Além disso, um teste “t” de "Student” foi realizado para detectar possíveis diferenças entre os materiais em relação aos tratamentos.

A análise dos dados indicou que houve efeito dos extratos da planta de alfafa sobre a germinação, o comprimento da radícula e do hipocótilo 
nos dois materiais testados. Apesar disso, o padrão de resposta, tanto em relação à parte da planta utilizada (raiz ou folhas) quanto às suas concentrações, mostrou-se diferente entre eles (Tabela 1). Apesar de os extratos aquosos das folhas terem inibido a germinação tanto do material Crioulo quanto da P5454, houve uma diferença importante na magnitude dos efeitos. A variedade P5454 foi mais sensível aos efeitos inibitórios na germinação que o material Crioulo (Tabela 1). Além disso, a germinação do material Crioulo foi significativamente superior $(\mathrm{P}=0,022)$ em relação à da variedade $\mathrm{P}-5454$, quando submetida aos extratos puros de folhas do material Crioulo. Estes resultados sugerem tolerâncias relativas importantes dentro de cada cultivar em relação aos princípios fitotóxicos.

A maior sensibilidade da variedade P5454 aos extratos da parte aérea pode estar relacionada com diferenças na tolerância aos compostos produzidos na própria planta. Como o extrato utilizado foi proveniente apenas do material Crioulo, é possível que esse material apresente uma tolerância maior aos aleloquímicos produzidos por ela mesma, razão pela qual a diluição F-50 praticamente não afetou sua germinação. Como não foram utilizados extratos da variedade P5454, não é possível determinar se ela também poderia ser mais tolerante aos extratos provenientes dela mesma.

Nos últimos anos, alguns esforços têm sido feitos no sentido de identificar os componentes químicos responsáveis pelos efeitos autotóxicos em alfafa. CHON \& KIM (2002) verificaram que os extratos das folhas causaram a maior redução no comprimento da raiz e do hipocótilo de alfafa e que os principais compostos envolvidos na inibição do desenvolvimento inicial da alfafa foram a cumarina, ácido trans-cinâmico e o ácido o-cumárico. Além disso, estes mesmos autores não detectaram a presença de tais compostos em amostras de raízes de alfafa, sugerindo que a ocorrência de efeito inibitório na germinação e no desenvolvimento das plântulas é resultado de compostos produzidos nas sementes, caules e folhas, principalmente.

Os comprimentos da radícula e do hipocótilo apresentaram resultados semelhantes àqueles observados para o percentual de germinação (Tabela 1). Interessante observar que os extratos puros das folhas reduziram significativamente a germinação dos dois materiais, sem que, no entanto, inibissem completamente sua germinação. Apesar disso, mesmo nas poucas sementes que germinaram não foi observado crescimento da radícula ou do hipocótilo. Alguns autores têm observado que os extratos afetam mais severamente o crescimento da radícula que o do hipocótilo (CHUNG \& MILLER, 1995; CHON et al., 2002). Apesar disso, os resultados encontrados no presente experimento não confirmaram os dados obtidos por esses autores, uma vez que os extratos (F50 e F100) afetaram de forma semelhante tanto o comprimento do hipocótilo quanto da radícula dos dois

Tabela 1 - Percentual de germinação, comprimento da radícula e hipocótilo (mm) da alfafa, material Crioulo e variedade P5454, submetida a extratos aquosos de raízes e folhas de alfafa, material Crioulo.

\begin{tabular}{lccc}
\hline Tratamentos $^{1}$ & Germinação (\%) & Comprimento da radícula (mm) & Comprimento do Hipocótilo (mm) \\
\hline & & Material Crioulo & 63,8A \\
Controle & $96,0 \mathrm{~A}$ & $53,5 \mathrm{~A}$ & $51,0 \mathrm{AB}$ \\
R-50 & $91,2 \mathrm{~A}$ & $44,8 \mathrm{~A}$ & $51,2 \mathrm{AB}$ \\
R-100 & $80,0 \mathrm{~A}$ & $45,0 \mathrm{~A}$ & $47,5 \mathrm{~B}$ \\
F-50 & $83,5 \mathrm{~A}$ & $42,1 \mathrm{~A}$ & $0,0 \mathrm{C}$ \\
F-100 & $6,4 \mathrm{~B}$ & $0,0 \mathrm{~B}$ & 42,7 \\
Média & 71,4 & 37,1 & 5,14 \\
EPM & 7,40 & 4,49 & \\
& & & $60,4 \mathrm{~A}$ \\
Controle & $74,4 \mathrm{~A}$ & $54,2 \mathrm{~A}$ & $53,4 \mathrm{~A}$ \\
R-50 & $69,2 \mathrm{~A}$ & $49,4 \mathrm{~A}$ & $44,2 \mathrm{~A}$ \\
R-100 & $65,6 \mathrm{~A}$ & $40,7 \mathrm{~A}$ & $25,6 \mathrm{~B}$ \\
F-50 & $38,0 \mathrm{~B}$ & $23,4 \mathrm{~B}$ & $0,0 \mathrm{C}$ \\
F-100 & $3,2 \mathrm{C}$ & $0,0 \mathrm{C}$ & 36,7 \\
Média & 50,1 & 33,5 & 3,28 \\
EPM & 4,17 & 3,52 & \\
\hline
\end{tabular}

${ }^{1}$ Controle: água deionizada; R-50: extrato da raiz diluído em 50\%; R-100: extrato puro da raiz; F-50: extrato das folhas diluído a 50\%; F100: extrato puro das folhas.

${ }^{2} \mathrm{EPM}=$ Erro padrão da média.

* Tratamentos com médias não seguidas pela mesma letra diferem pelo teste de Tukey a 5\% de probabilidade de erro.

Ciência Rural, v.38, n.2, mar-abr, 2008. 
materiais (Tabela 1), sugerindo existirem também diferenças varietais quanto ao local preferencial de inibição na planta.

É importante salientar que o poder inibitório de extratos de plantas sobre outras plantas, detectado por meio de ensaios de laboratório, não indica necessariamente a ocorrência de efeitos alelopáticos sob condições de campo. Segundo RICE (1984), uma parte essencial da alelopatia é o movimento do agente alelopático potencial no solo. A natureza química desses produtos é muito diversa e alguns só atuam quando em presença de outros, em combinações e proporções específicas, sendo difícil distinguir e identificar os efeitos individuais, devido à complexidade biológica do processo (ALMEIDA, 1988). De acordo com JENNINGS \& NELSON (2002b), os compostos aleloquímicos no solo possuem uma zona de interferência sobre a germinação e o desenvolvimento inicial da alfafa de aproximadamente 20 a $25 \mathrm{~cm}$, indicando que resultados obtidos em experimentos de laboratório devem ser interpretados com cautela. Dessa forma, apesar de os dados obtidos no presente experimento evidenciarem potencial autotóxico de extratos de folhas sobre a germinação e o desenvolvimento inicial da cultura de alfafa, é necessária a realização de estudos de campo, com o objetivo de determinar se variáveis como longevidade do estande, época do ano, tempo de retardo na (re)semeadura e a longevidade de ação dos compostos inibitórios no solo apresentam efeitos inibitórios significativos sobre a cultura, em condições de campo.

Os resultados apresentados no presente trabalho sugerem que há indícios de sensibilidade intra-específica na inibição da germinação e no crescimento de raízes e hipocótilos de alfafa, em que extratos de folhas podem ser mais prejudiciais ao desenvolvimento de outros materiais que a ela própria. No entanto, pesquisas mais detalhadas são necessárias, com materiais diversos, a fim de confirmar esta hipótese.

\section{REFERÊNCIAS}

ALMEIDA, F.S. A alelopatia e as plantas. IAPAR: Londrina, PR, 1988. 60p. (Circular n.53).

CHON, S.U. et al. Effects of alfalfa leaf extracts and phenolic allelochemicals on early seedling growth and root morphology of alfalfa and barnyard grass. Crop Protection, v.21, n.10, 1077-1082, 2002.
CHON, S.U.; KIM, J.D. Biological activity and quantification of suspected allelochemicals from alfalfa plant parts. Journal of Agronomy and Crop Science, v.188, n.4, p.281-285, 2002.

CHON, S.U. et al. Physiological assessment and path coefficient analysis to improve evaluation of alfalfa autotoxicity. Journal of Chemical Ecology, v.29, n.11, p.2413-2424, 2003.

CHON, S.U. Allelopathic and autotoxic effects of alfalfa plant and soil extracts. Korean Journal of Crop Science, v.49, n.1, p.7-11, 2004.

CHON, S.U. et al. Alfalfa (Medicago sativa L.) autotoxicity: Current status. Allelopathy Journal, v.18, n.1, p.57-80, 2006.

CHUNG, I.M.; MILLER, D.A. Effect of alfalfa plant and soil extracts on germination and growth of alfalfa. Agronomy Journal, v.87, n.4, p.762-767, 1995.

JENNINGS, J.A.; NELSON, C.J. Zone of autotoxic influence around established alfalfa plants. Agronomy Journal, v.94, n.5, p.1104-1111, 2002a.

JENNINGS, J.A.; NELSON, C.J. Rotation interval and pesticide effects on establishment of alfalfa after alfalfa. Agronomy Journal, v.94, n.4, p.786-791, 2002b.

JENSEN, E.H. et al. Autotoxicity in alfalfa. Reno, NY: University of Nevada, 1981. (UNIVERSITY OF NEVADA AGRICULTURAL EXPERIMENTAL STATION BULLETIN, R.144).

KONO, M. et al. Studies on allelopathy in barley (Hordeum vulgare L.). Japanese Journal of Crop Science, v.68, p.419423, 1999.

MILLER, D.A. Allelopathy in forage crop systems. Agronomy Journal, v.88, n.6, p.854-859, 1996.

NUERNBERG, N.J. Técnicas de produção de alfafa. In: PEIXOTO, A.M. et al. Pastagens: fundamentos da exploração racional. Piracicaba: Fealq, 1994. p.657-678.

PUTNAM, A.R.; DUKE, W.B. Biological suppression of weeds -evidence for allelopathy in accessions of cucumber. Science, v.185, n.4148, p.370-372, 1974.

RASSINI, J.B. et al. Cultivo da alfafa. 2003. Capturado em 20 set. 2006. Online. Disponível na Internet http:// sistemasdeproducao.cnptia.embrapa.br/FontesHTML/ Alfafa/ SistemaProducaoAlfafa.

RICE, E.L. Allelopathy. New York: Academic, 1984. 422p.

XUAN, T.D.; TSUZUKI, E. Varietal differences in allelopathic potential of alfalfa. Journal of Agronomy and Crop Science, v.188, n.1, p.2-7, 2002. 